\title{
Production of nonulosonic acids in the extracellular polymeric substances of "Candidatus Accumulibacter phosphatis"
}

\author{
Sergio Tomás-Martínez ${ }^{1}$ (D) Hugo B.C. Kleikamp ${ }^{1}$ (D) Thomas R. Neu $^{2}$ (D) Martin Pabst $^{1}$ (D) David G. Weissbrodt $^{1}$ (D) \\ Mark C.M. van Loosdrecht ${ }^{1}$ (D) Yuemei Lin $^{1}$ (i)
}

Received: 3 November 2020 / Revised: 2 February 2021 / Accepted: 17 March 2021 / Published online: 1 April 2021

(C) The Author(s) 2021

\begin{abstract}
Nonulosonic acids (NulOs) are a family of acidic carbohydrates with a nine-carbon backbone, which include different related structures, such as sialic acids. They have mainly been studied for their relevance in animal cells and pathogenic bacteria. Recently, sialic acids have been discovered as an important compound in the extracellular matrix of virtually all microbial life and in "Candidatus Accumulibacter phosphatis", a well-studied polyphosphate-accumulating organism, in particular. Here, bioaggregates highly enriched with these bacteria (approx. 95\% based on proteomic data) were used to study the production of NulOs in an enrichment of this microorganism. Fluorescence lectin-binding analysis, enzymatic quantification, and mass spectrometry were used to analyze the different NulOs present, showing a wide distribution and variety of these carbohydrates, such as sialic acids and bacterial NulOs, in the bioaggregates. Phylogenetic analysis confirmed the potential of " $\mathrm{Ca}$. Accumulibacter" to produce different types of NulOs. Proteomic analysis showed the ability of " $\mathrm{Ca}$. Accumulibacter" to reutilize and reincorporate these carbohydrates. This investigation points out the importance of diverse NulOs in non-pathogenic bacteria, which are normally overlooked. Sialic acids and other NulOs should be further investigated for their role in the ecology of " $\mathrm{Ca}$. Accumulibacter" in particular, and biofilms in general.
\end{abstract}

\section{Key Points}

- "Ca. Accumulibacter" has the potential to produce a range of nonulosonic acids.

-Mass spectrometry and lectin binding can reveal the presence and location of nonulosonic acids.

-The role of nonulosonic acid in non-pathogenic bacteria needs to be studied in detail.

Keywords Nonulosonic acids · "Candidatus Accumulibacter phosphatis" - Sialic acids · Extracellular polymeric substances · Granular sludge $\cdot$ Biological phosphate removal

\section{Introduction}

Wastewater transports polluting nutrients, such as organic matter, phosphorus $(\mathrm{P})$, or nitrogen $(\mathrm{N})$. When $\mathrm{P}$ and/or $\mathrm{N}$ are in excess, discharging this wastewater into surface

Sergio Tomás-Martínez

S.TomasMartinez@tudelft.nl

1 Department of Biotechnology, Delft University of Technology, Van der Maasweg 9, 2629 HZ, Delft, The Netherlands

2 Microbiology of Interfaces, Department River Ecology, Helmholtz Centre of Environmental Research - UFZ, Brueckstrasse 3A, 39114 Magdeburg, Germany waters leads to eutrophication. Thus, these pollutants must be eliminated from wastewater streams (Mainstone and Parr 2002). Enhanced biological phosphorus removal (EBPR) has become a widely applied treatment to eliminate inorganic phosphorus and organic matter from wastewater. This technology exploits the metabolic capacity of polyphosphate-accumulating organisms (PAOs) to take up inorganic phosphorus and to store it in the form of intracellular polyphosphate. "Candidatus Accumulibacter phosphatis", a well-studied model PAO, has been identified as a dominant species responsible for EBPR (Seviour et al. 2003). This microorganism has not been isolated yet. It grows in the form of compact microcolonies and bioaggregates (flocs, granules, or biofilms) held together 
by extracellular polymeric substances (EPS) (Weissbrodt et al. 2013; Barr et al. 2016).

EPS is a complex mixture of biopolymers of different nature, such as polysaccharides, proteins, nucleic acids, or lipids, among others. These biopolymers are synthesized or released by microorganisms across their life cycle, forming matrices that provide mechanical stability and act as a scaffold for the microorganisms in biofilms (Flemming and Wingender 2010). Although research in the past years led to analytical advances for the extraction and characterization of EPS (Felz et al. 2016; Boleij et al. 2018; Felz et al. 2019; Boleij et al. 2019), the EPS matrix still represents the "dark matter" of biofilms that need to be studied in more detail (Neu and Lawrence 2016; Neu and Lawrence 2017; Seviour et al. 2019). The pragmatic study of individual components (i.e., proteins and carbohydrates like monosaccharides and polysaccharides) can give new insights into the understanding of EPS as a whole. Recently, sialic acids have been detected and described in the EPS of both EBPR and salt-adapted aerobic granular sludge with the presence of " $\mathrm{Ca}$. Accumulibacter" using fluorescence lectin-binding analysis (FLBA) coupled to confocal laser scanning microscopy (CLSM) (Weissbrodt et al. 2013; de Graaff et al. 2019).

Sialic acids are a subset of a family of $\alpha$-keto acids with a nine-carbon backbone, called nonulosonic acids (NulOs). These carbohydrates are unusual among the various monosaccharide building blocks of extracellular glycoconjugates, which normally have five or six carbons. Sialic acids are typically found as terminal residues on the glycan chains of vertebrate extracellular glycoconjugates, making them the "bridging" or recognition molecules between cells, as well as between cells and extracellular matrix (Chen and Varki 2010). The distinct features of sialic acids contribute to higher structural complexity and the potential for more unique and varied biological functions, in comparison to other monosaccharides (Deng et al. 2013).

Looking into the specific chemical structure, sialic acids are derivatives of neuraminic (Neu) and ketodeoxynonulosonic (Kdn) acids. The most studied one is $\mathrm{N}$-acetylneuraminic acid (Neu5Ac) (Varki et al. 2017). Apart from these acids, other NulOs have been found only in microbes, such as the isomers pseudaminic (Pse) and legionaminic (Leg) acids, which are structurally similar to sialic acids (Fig. 1a) (Knirel et al. 2003). These NulOs have recently been described as ubiquitous in the microbial world (Lewis et al. 2009; Kleikamp et al. 2020a), making their study and understanding highly relevant.

Despite the different chemical structure, the NulOs share similarities in their metabolic pathway (Fig. 1c). The common steps in each NulO biosynthetic pathway (NAB) are catalyzed by homologous enzymes: the condensation of a 6-carbon intermediate with phosphoenol pyruvate (PEP) produces a 9-C $\alpha$-keto acid (catalyzed by the enzyme NAB-2); its activation results from the addition of CMP by the enzyme NAB-1 (Lewis et al. 2009). The different NulOs can be further modified by additional substitutions on the hydroxyl groups such as $O$-acetyl, $O$-methyl, $O$-sulfate, $O$-hydroxybutyryl, $O$-formyl, or $O$-lactyl groups (Fig. 1b) (Angata and Varki 2002). A phylogenetic analysis of the NAB-2 enzyme, the most conserved one in the pathway, can be used to predict the NulO types synthesized by an organism (Lewis et al. 2009). In the case of the sialic acids NeuAc and Kdn, the same biosynthetic machinery leads to their synthesis, using $N$ acetylmannosamine or mannose as a starting substrate respectively (Varki et al. 2017).

Investigations of NulOs have been predominantly focused in animal cells and pathogenic bacteria. Among the large diversified NulOs, Neu5Ac is often assumed as the most dominant one present in biological samples. It plays important roles in recognition processes or stabilization of biomolecules (Hanisch et al. 2013). In animals, it is crucial to physiological processes, such as recognition between cells and neuronal transmission, or diseases such as cancer and autoimmune diseases (Traving and Schauer 1998). In pathogenic bacteria, NulOs contribute in delaying the host's immune response by mimicking the host's glycosylation pattern (Carlin et al. 2009).

Regardless of the intensive studies of NulOs in animal tissue and on the surface-related structure of pathogenic bacteria cells, the presence, production, and function of NulOs in non-pathogenic bacteria have not been widely realized and studied. Only very recently, a genome-level study (Lewis et al. 2009) and a NulO universal survey by high-resolution mass spectrometry (Kleikamp et al. 2020a) revealed the unexpectedly wide distribution of nonulosonic acid biosynthesis (NAB) pathway genes and widespread occurrence of NulOs in non-pathogenic bacteria. These discoveries indicate that NulOs must be an important component in the EPS of bacterial aggregates in natural and engineered ecosystems, which has been completely overlooked at present. It also indicates that the current model of evolution and utilization of sialic acids in prokaryotes which is driven by host-pathogen interactions may not reflect the complete picture and need to be questioned (Lewis et al. 2009; Kleikamp et al. 2020a).

It is known that " $\mathrm{Ca}$. Accumulibacter" is not only the most abundant and well-studied PAO in EBPR systems but also contributes to phosphate sequestration and phosphate cycling in estuarine systems (Watson et al. 2019). It is unknown how many types of NulOs can be produced and what the potential pathways are. Studying the diversity, production, and utilization of NulOs with an enriched culture of " $\mathrm{Ca}$. Accumulibacter" will add new information to the ecology of this important microorganism, i.e., if diverse NulO production is one of the strategies to win 


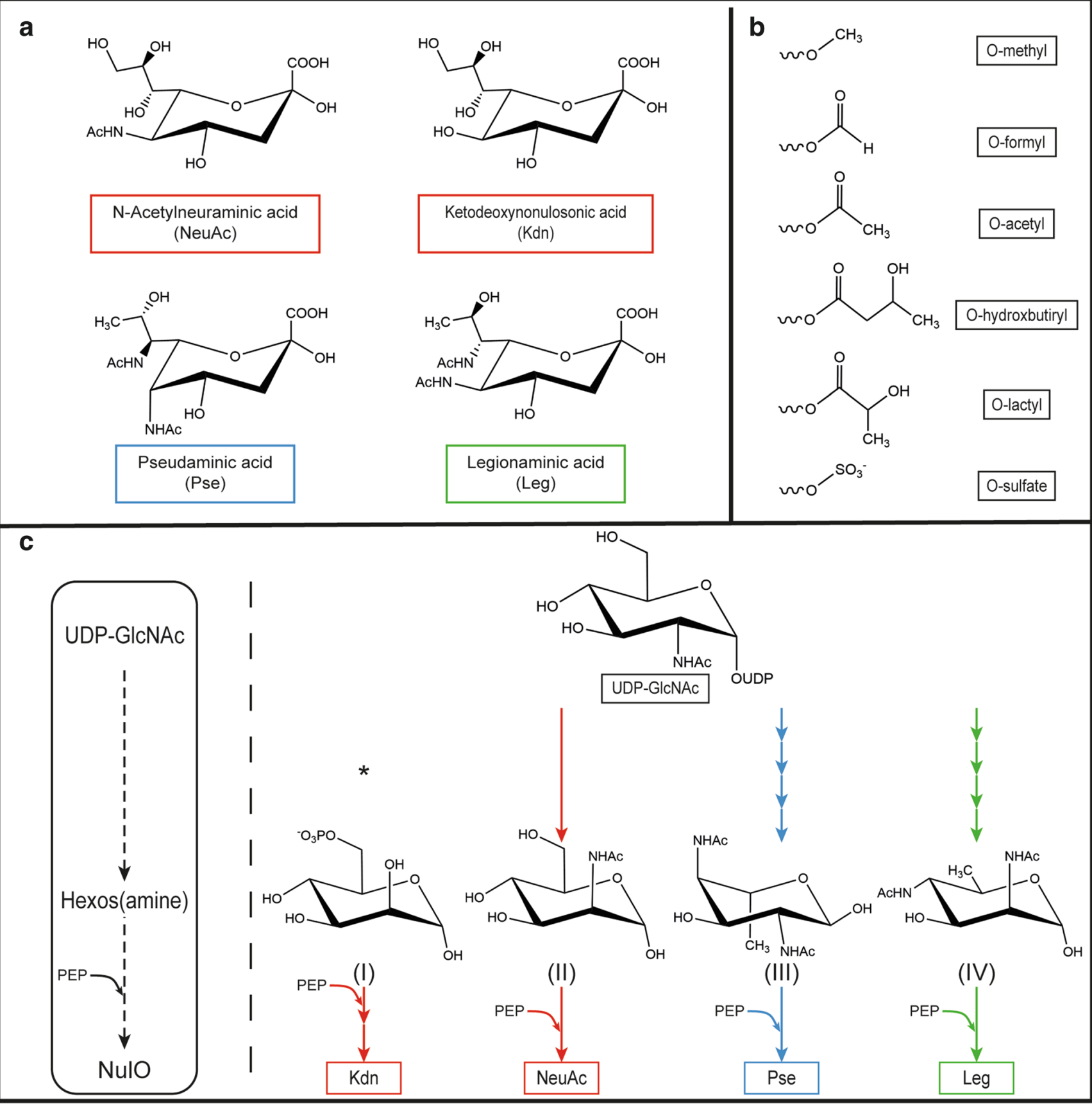

Fig. 1 Common metabolic pathway for the biosynthesis of different NulOs. a Chemical structure of different NulOs. b Possible modifications of the hydroxyl groups NulOs. c Core (left) and specific (right) biosynthetic pathways for the different NulOs. The biosynthetic pathways of the different NulOs branch from UDP-GlcNAc, with the

the competition over other microorganisms in the system. Furthermore, it will provide valuable insights into the synthesis and turnover of NulOs (or sialic acids) by nonpathogenic environmental bacteria. The study of the role of NulOs outside the pathogen-host interaction will extend the current understanding of ecology and evolution of these carbohydrates. exception of Kdn. Each arrow represents one enzymatic step. NeuAc and $\mathrm{Kdn}$ share the enzymes involved in the synthesis. (I) mannose-6phosphate; (II) N-acetylmannosamine; (III) 2,4-diacetamido-2,4,6trideoxy-L-altropyranose; (IV) 2,4-diacetamido-2,4,6-trideoxy-dmannopyranose. Adapted from (Lewis et al. 2009)

The objective of this research is to confirm the presence, predict the production, and explore the diversity of NulOs in "Ca. Accumulibacter". To achieve this, " $\mathrm{Ca}$. Accumulibacter" was enriched using a lab-scale sequencing batch reactor with EBPR performance (Guedes da Silva 2020). NulOs produced by this biomass were analyzed by a combination of techniques, such as fluorescence lectin- 
binding analysis (FLBA), enzymatic release, and mass spectrometry. Genomic and proteomic investigations were conducted to evaluate the diversity of pathways involved in $\mathrm{NulO}$ formation and utilization by " $\mathrm{Ca}$. Accumulibacter".

\section{Materials and methods}

\section{"Ca. Accumulibacter"-enriched biomass and seawater-adapted aerobic granules}

An in-house enrichment culture of " $\mathrm{Ca}$. Accumulibacter" was used (Guedes da Silva 2020). The enrichment was maintained in a 1.5-L sequencing batch reactor (SBR), with slight modifications from the SBR-2 described in Guedes da Silva et al. (2018). The COD-based acetate to propionate ratio in the feed was 65:35 $\mathrm{g}_{\mathrm{COD}} / \mathrm{g}_{\mathrm{COD}}$. Fluorescence in situ hybridization (FISH) showed the dominance of PAO in the system (approx. 95\% of biovolume), and 16S rRNA gene amplicon sequencing confirmed " $\mathrm{Ca}$. Accumulibacter" as the dominant PAO. Proteomic investigations by Kleikamp et al. (2020b) further confirmed this dominance (approx. 95\%). Seawater-adapted aerobic granules from de Graaff et al. (2019) were also used in the study. FISH showed the dominance of PAO in these granules as well.

\section{Nonulosonic acid analyses}

\section{Fluorescence lectin-binding analysis (FLBA)}

Lectin staining of the biomass was done according to earlier works (Weissbrodt et al. 2013; Boleij et al. 2018; de Graaff et al. 2019). Bioaggregates enriched with " $C a$. Accumulibacter" were stained and mounted in coverwell chambers with a $0.5-\mathrm{mm}$ spacer in order to avoid squeezing of the samples. Glycoconjugates of the biomass were examined by means of barcoding with green fluorescent lectins (Neu and Kuhlicke 2017). Thus, all commercially available lectins labelled with a green fluorophore (FITC or Alexa488) were applied as probes individually to different aggregates. A total of 77 lectins were used to screen glycoconjugates (Bennke et al. 2013). The binding sites of the sialic acidspecific lectins that gave the strongest signal are listed in Table 1. After incubation with the lectin solution, the sample was washed with tap water for three times in order to remove unbound lectins. For 3D imaging, a TCS SP5X confocal laser scanning microscope (Leica, Germany) was employed. The system comprised an upright microscope and a super continuum light source (white laser). The hardware setup was controlled by the software LAS AF 2.4.1. Confocal data sets were recorded by using $25 \times$ NA 0.95 and $63 \times$ NA 1.2 water immersion lenses. Excitation was at $490 \mathrm{~nm}$ and emission signals were detected simultaneously with two photomultipliers from 480 to $500 \mathrm{~nm}$ (reflection) and 505-580 nm (fluorescence). Image data sets were deconvolved with Huygens version 18.04 using blind deconvolution (SVI, The Netherlands) and projected with Imaris version 9.2 (Bitplane, Switzerland). Images were printed from Photoshop CS6 (Adobe).

\section{Nonulosonic acid diversity and enzymatic quantification}

The diversity of NulOs in bioaggregates from the " $\mathrm{Ca}$. Accumulibacter" enrichment and in seawater-adapted aerobic granules (de Graaff et al. 2019) was analyzed by highresolution mass spectrometry according to Kleikamp et al. (2020a), with the addition of manual verification of lower abundant species. The Sialic Acid Quantitation Kit (SigmaAldrich, USA) was used to estimate the content of sialic acids (Neu5Ac as model one) in the enriched " $\mathrm{Ca}$. Accumulibacter" biomass following the manual instructions. A detailed description of the protocol can be found in the Supplementary methods.

\section{Genomic analysis of pathways for biosynthesis of different nonulosonic acids}

\section{BLAST (Basic Local Alignment Search Tool) analysis of key enzymes}

In order to predict the potential production of different NulOs by " $\mathrm{Ca}$. Accumulibacter", different near-complete draft metagenome-assembled genomes (MAGs) of " $\mathrm{Ca}$. Accumulibacter" (Rubio-Rincón et al. 2019) were studied. These MAGs were used to get the amino acid sequences of nonulosonic acid synthases (NAB-2), i.e., the most conserved enzyme of the biosynthetic pathway, which condensates a 6carbon intermediate with pyruvate to produce a 9-carbon $\alpha$ -

Table 1 Sialic acid-specific lectin used in this analysis

\begin{tabular}{llll}
\hline Lectin name & Abbreviation & Ligand motif & Reference \\
\hline Cancer antennarius lectin & CCA & 9-O-Ac-NeuAc; 4-O-Ac-NeuAc & Ravindranath et al. (1985) \\
Maackia amurensis lectin & MAA & Neu5Ac $(\alpha 2-3)$ Gal( $\beta 1-4) G l c N a c / G l c$ & Knibbs et al. (1991) \\
Sambucus nigra lectin & SNA & Neu5Ac $(\alpha 2-6) G a l / G a l N A c$ & Shibuya et al. (1987) \\
Wheat germ agglutinin & WGA & Internal GlcNAc; Neu5Ac & Gallagher et al. (1985) \\
\hline
\end{tabular}


keto acid (Fig. 1c). A protein sequence alignment versus a protein database (BLASTp), or a protein sequence alignment versus a translated nucleotide sequence database (TBLASTn; when only nucleotide sequences were available) from the NCBI website (blast.ncbi.nlm.nih.gov/Blast.cgi) was performed using the sequence of the known $\mathrm{N}$ acetylneuraminic acid synthase from Campylobacter jejuni (accession number: CAL35431.1) as query. E-values lower than $5 \mathrm{e}-15$ were set as positive result and chosen for the phylogenetic analysis (Petit et al. 2018).

\section{Phylogenetic analysis of NAB-2 sequences}

In order to predict the specific NulO synthesized by the different NAB-2 enzymes from the MAGs of " $\mathrm{Ca}$. Accumulibacter", the phylogenetic method developed by Lewis et al. (2009) was performed. For this analysis, NAB2 amino acid sequences with known specificity from different bacteria, archaea, and animals were employed. Less conserved enzymes from "Ca. Accumulibacter" (Table 2 $\mathrm{M}-\mathrm{S}$ ) were removed from the analysis in order to improve the final alignment. The removed sequences might represent unknown specificities not included in the analysis (Lewis et al. 2009).

Table 2 Selected NAB-2 enzymes (nonulosonic acid synthase) from "Ca. Accumulibacter" used for the phylogenetic analysis. Amino acid sequences were obtained by performing BLASTp using the NAB-2 enzyme from $C$. jejuni (accession number: CAL35431.1) as query. No accession numbers are provided for the enzymes where no protein

\section{Shotgun proteomic analysis}

Shotgun proteomic analysis of the "Ca. Accumulibacter" enrichment was performed as described in the study of Kleikamp et al. (2020b). A detailed description of the methodology can be found in the Supplementary methods.

\section{Results}

The enrichment culture of " $\mathrm{Ca}$. Accumulibacter" was derived from the system described by Guedes da Silva (2020). Data describing the performance of the enrichment are given by the authors. As shown by FISH and proteomic data (Kleikamp et al. 2020b), the bioaggregates used in this research were highly enriched with " $\mathrm{Ca}$. Accumulibacter" (approx. 95\%).

\section{Nonulosonic acid analyses}

\section{Fluorescence lectin-binding analysis (FLBA)}

Lectins are proteins that bind to specific carbohydrate groups. Fluorescence-labelled lectins can be used as probes for the in situ analysis of glycoconjugates in the EPS of bioaggregates

sequences were available as the whole nucleotide sequence was used. Genomes were recovered from on-line public databases such as NIH GenBank (GCA accession numbers), NCBI RefSeq (GCF accession numbers), and JCI MGM ( $G a$ accession numbers)

\begin{tabular}{|c|c|c|c|c|}
\hline Enzyme reference & Enzyme accession number & E-value & Genome name & Genome accession number \\
\hline A & 2626517415 & $6.00 \mathrm{E}-53$ & “Ca. Accumulibacter sp. UW-2" & $\mathrm{Ga} 0078784$ \\
\hline $\mathrm{B}$ & 2689798196 & $7.00 \mathrm{E}-53$ & "Ca. Accumulibacter phosphatis Type IA UW-3" & $\mathrm{Ga} 0131788$ \\
\hline $\mathrm{C}$ & 2626510243 & $7.00 \mathrm{E}-53$ & “Ca. Accumulibacter sp. UW-?" & $\mathrm{Ga} 0078783$ \\
\hline $\mathrm{D}$ & OJW49354.1 & $2.00 \mathrm{E}-49$ & "Ca. Accumulibacter sp. 66-26" & GCA 001897745 \\
\hline $\mathrm{E}$ & KFB70901.1 & $3.00 \mathrm{E}-49$ & "Ca. Accumulibacter sp. BA-91" & GCF 000585035 \\
\hline $\mathrm{F}$ & OJW47994.1 & $2.00 \mathrm{E}-42$ & "Ca. Accumulibacter sp. 66-26" & GCA 001897745 \\
\hline G & KFB74173.1 & $5.00 \mathrm{E}-40$ & "Ca. Accumulibacter sp. BA-91" & GCF 000585035 \\
\hline $\mathrm{H}$ & KFB66819.1 & $6.00 \mathrm{E}-39$ & "Ca. Accumulibacter sp. SK-01" & GCA 000584955 \\
\hline I & KFB76891.1 & $1.00 \mathrm{E}-38$ & "Ca. Accumulibacter sp. SK-02" & GCA 000584975 \\
\hline $\mathrm{J}$ & WP_046535243.1 & $3.00 \mathrm{E}-38$ & "Ca. Accumulibacter phosphatis UW-1" & GCA 000024165 \\
\hline K & - & $7.00 \mathrm{E}-38$ & “Ca. Accumulibacter sp. UBA 5574” & GCA 002425405 \\
\hline $\mathrm{L}$ & - & $2.00 \mathrm{E}-37$ & “Ca. Accumulibacter sp. HKU-2” & GCF 000987395 \\
\hline M & HCZ15346.1 & $5.00 \mathrm{E}-22$ & “Ca. Accumulibacter sp. UBA11064" & GCA 003538495 \\
\hline $\mathrm{N}$ & HCN68329.1 & $2.00 \mathrm{E}-21$ & “Ca. Accumulibacter sp. UBA 11070" & GCA 003535635 \\
\hline $\mathrm{O}$ & EXI67255.1 & $7.00 \mathrm{E}-21$ & “Ca. Accumulibacter sp. SK-12” & GCA 000585015 \\
\hline $\mathrm{P}$ & - & $3.00 \mathrm{E}-20$ & “Ca. Accumulibacter sp. UBA 2783" & GCA 002352265 \\
\hline Q & EXI83869.1 & $6.00 \mathrm{E}-20$ & "Ca. Accumulibacter sp. BA-94" & GCA 000585095 \\
\hline $\mathrm{R}$ & EXI78685.1 & $7.00 \mathrm{E}-20$ & “Ca. Accumulibacter sp. BA-92” & GCA 000585055 \\
\hline S & RDE52394.1 & $5.00 \mathrm{E}-19$ & "Ca. Accumulibacter sp. UW-LDO-IC" & GCA 003332265 \\
\hline
\end{tabular}


(Neu and Kuhlicke 2017). Intact biomass samples collected from the "Ca. Accumulibacter" enrichment culture were screened with 77 lectins (data not shown). Some sialic acidspecific lectins gave a positive result, such as CCA, WGA, MAA, and SNA (the binding site of these lectins can be found in Table 1). Especially, MAA and SNA gave the strongest signal (Fig. 2a, b). The signals from both lectins were widely distributed across the aggregate, mainly detected at the surface of the bacterial cells (a more detailed view can be seen in Fig. $3)$. SNA recognizes $\alpha-2,6$-linked sialic acid, while MAA recognizes $\alpha$-2,3-linked sialic acid (Soares et al. 2000) (Table 1). The strong signals from both SNA and MAA lectins indicated that the sialic acids on the cell surface of " $\mathrm{Ca}$. Accumulibacter" present both types of linkages. In contrast with SNA and MAA, the distribution of the signal of WGA showed the presence of lectin-specific glycoconjugates in other parts of the biomass (Fig. 2c). This may be due to the wider specificity of WGA (e.g., towards GlcNAc). In addition, although CCA gave a low signal (Fig. 2d), it indicated the presence of sialic acids with different modifications, as it is specific for the staining of 9-O or 4-O-acetyl NeuAc.

\section{Nonulosonic acid diversity by mass spectrometry analysis}

Lectin staining showed a wide distribution of different sialic acids in the " $\mathrm{Ca}$. Accumulibacter"-enriched bioaggregates.
However for this, not only the type of NulO but also the same motifs (linkage type and sub-terminal monosaccharide) can result in binding. In order to fully explore the diversity of NulOs, manual verification was added in mass spectrometry analysis, which allowed the detection of relatively lower abundant species. Similar as what was reported in Kleikamp et al. (2020a): bacterial NulOs (Pse/Leg) were found dominant both in the enrichment and seawater-adapted aerobic granular sludge, NeuAc was only detected in the latter biomass. Unlike Kleikamp et al. (2020a), a small amount of Kdn was detected in the enrichment. Since both samples were enriched with "Ca. Accumulibacter", it seems that bacterial NulOs (Pse/ Leg), NeuAc, and Kdn might be the potential NulOs that this microorganism can produce.

\section{Sialic acid enzymatic release}

Bacterial NulOs and sialic acids are widely distributed in the enriched " $\mathrm{Ca}$. Accumulibacter" biomass. In order to quantify the amount of sialic acids present in the enrichment, a commercial enzymatic assay was performed, using a broadspectrum sialidase $(\alpha-(2 \rightarrow 3,6,8,9)$-neuraminidase $)$. The enzyme releases $\alpha-2,3-, \alpha-2,6-, \alpha-2,8-$, and $\alpha-2,9-$ linked NeuAc. The liberated sialic acids are then detected and quantified after a reaction with an aldolase and dehydrogenase. Unfortunately, no sialic acids were released. This could be
Fig. 2 Confocal laser scanning microscopy (CLSM) after fluorescence lectin-binding analysis (FLBA). Images show bioaggregates enriched in " $\mathrm{Ca}$. Accumulibacter". The glycoconjugates visualized in a-d show four different sialic acidspecific lectins (a MAA, b SNA, c WGA, d CCA). The reflection signals either mark reflective particles associated with the granules $(\mathbf{a}, \mathbf{b})$ or outline the shape of the granule due to cell internal reflections $(\mathbf{c}, \mathbf{d})$. Color allocation: greenglycoconjugates, whitereflection signal
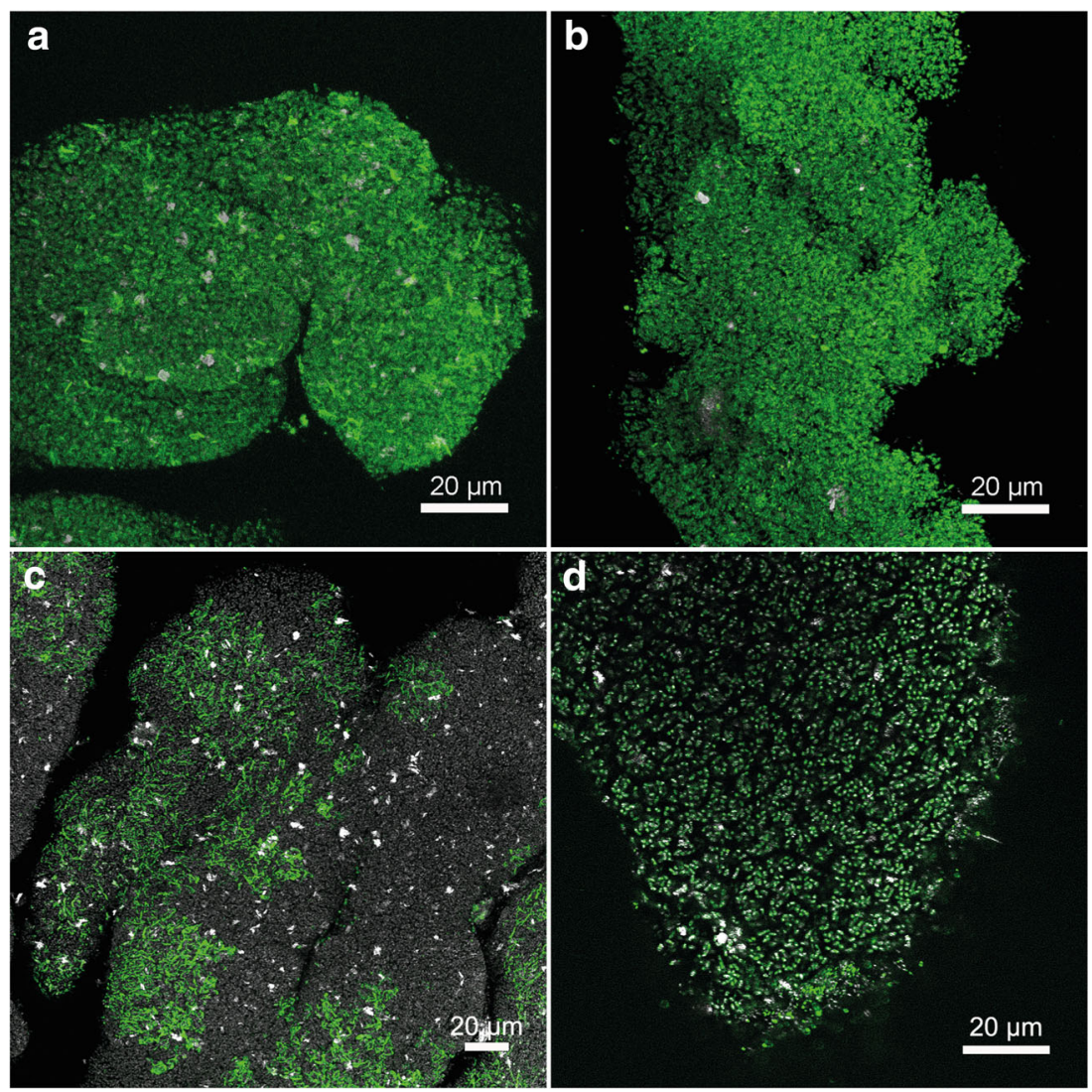
Fig. 3 Zoomed in section of a granule showing more details of glycoconjugate distribution after lectin staining. The settings for projections were defined in a way that strong and weak signals can be differentiated in different colors. a Strong signal of lectin MAA at the surface of the bacterial cells (shown in green). Please take notice of the blob-like appearance of the

glycoconjugates at the bacterial cell surface. b Weak signal of the lectin MAA in the space in between bacterial cells (shown in red). $\mathbf{c}$ Overlay of $\mathbf{a}$ and $\mathbf{b}$. $\mathbf{d}-\mathbf{f}$

The same is shown for the lectin SNA
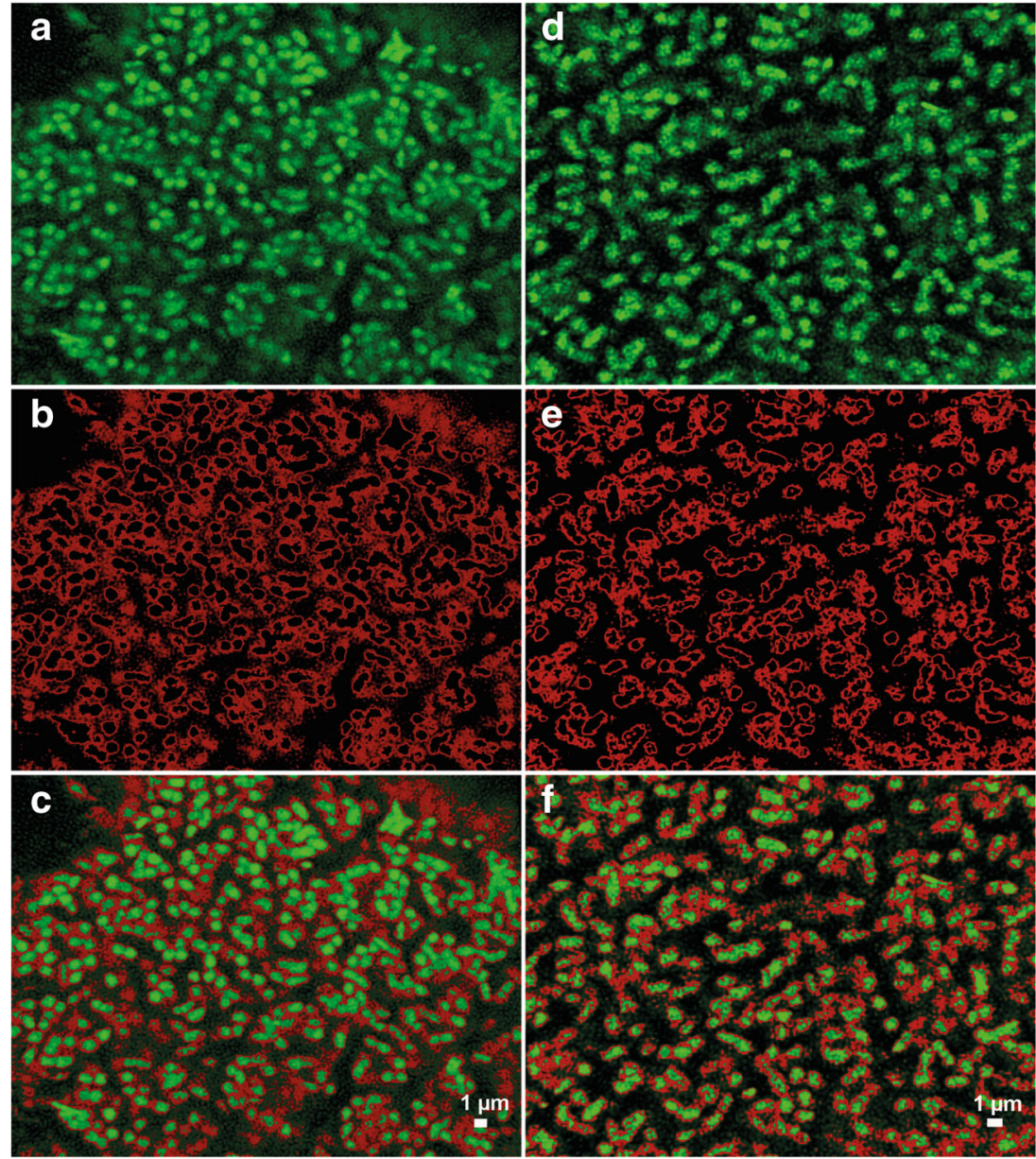

due to the specificity of the sialidase which might recognize only NeuAc, but not other variants of sialic acids (e.g., Kdn) and bacterial NulOs. According to MS analysis, NeuAc was present in seawater-adapted aerobic granules, but not in the enriched " $\mathrm{Ca}$. Accumulibacter" biomass, which explains why the amount of sialic acids was successfully quantified by the enzymatic assay in seawater-adapted granules as described in de Graaff et al. (2019), but was unsuccessful for " $C a$. Accumulibacter" enrichment. In fact, sialidases have been reported to differ in their sensitivity; e.g., it was found that Kdn is linked to almost all glycan structures in place of NeuAc, but it has lower sensitivity to sialidase which is specific for NeuAc (Lambre et al. 1982).

\section{Phylogenetic analysis}

Various metagenome-assembled genomes (MAGs) of “ $\mathrm{Ca}$. Accumulibacter" available in public repositories and surveyed in literature (Rubio-Rincón et al. 2019) were used to predict the potential diversity of NulOs that " $\mathrm{Ca}$. Accumulibacter" can produce. The prediction was focused on the NulO synthase (NAB-2), the enzyme that condenses a 6-carbon intermediate with phosphoenol pyruvate to yield a 9 -carbon $\alpha$-keto acid. This is a common step in the biosynthetic pathway of the different NulOs and the most conserved enzyme in the metabolic route (Lewis et al. 2009). The enzyme NeuAc synthase from Campylobacter jejuni (accession number: CAL35431.1) was used to obtain NAB-2 amino acid sequences from the different genomes of "Ca. Accumulibacter" (Table 2). Different reported NAB-2 amino acid sequences were used as query, giving similar results (data not shown). All the potential NAB-2 enzymes listed present low e-value, ranging from $6 \mathrm{e}-53$ to $5 \mathrm{e}-19$. Although the NeuAc synthase from $C$. jejuni matched with most of the available MAGs of " $\mathrm{Ca}$. Accumulibacter", some of them did not show the presence of this enzyme, which can be both due to a poor assembly or annotation of the genome or to the genetic incapacity of some genotypes to produce NulOs.

These amino acid sequences were used to predict their potential specificity using the phylogenetic analysis method developed by Lewis et al. (2009). The sequences of " $\mathrm{Ca}$. Accumulibacter" with the higher e-values (M-S in Table 2) were eliminated from the analysis as they appeared to be lessconserved and affected the multiple alignment and therefore the phylogenetic analysis. These divergent sequences might indicate specificity for a different $\mathrm{NulO}$ than the ones used for 
the final analysis (Lewis et al. 2009). The rest of the enzymes of " $\mathrm{Ca}$. Accumulibacter", together with sequences from animal, bacteria, and archaea, were used to generate a distancebased neighbor-joining tree (Fig. 4).

The different NAB-2 sequences were grouped based on their predicted specificities. Four groups are generated corresponding to legionaminic acid (Leg), pseudaminic acid (Pse), neuraminic acid ( $\mathrm{Neu}$ ), and "animal-like" NulOs, which reflects a novel phylogenetic class for which no biochemical data currently exist (Lewis et al. 2009). Within these four groups, NAB-2 enzymes from "Ca. Accumulibacter" were located in the three groups: Pse, Leg, and Neu. For the genomes that presented more than one copy of NAB-2, each of the copy was predicted to produce either the sialic acid Neu or the one of the bacterial structures (Pse/Leg). Therefore, it is predicted that " $\mathrm{Ca}$. Accumulibacter" has the potential to synthesize NulOs with one or two different core structures (Pse/Leg and Neu). Looking back at the mass spectrometry (MS) results, the enriched " $\mathrm{Ca}$. Accumulibacter" in this research produced bacterial NulOs (as Leg and Pse are isomers, they cannot be differentiated by MS analysis) and $\mathrm{Neu}$ (including Kdn and derived forms of Neu (e.g., $\mathrm{NeuAc}$ ) as well since they share the same pathway with $\mathrm{Neu}$, which is in consistence with the phylogenetic prediction.

\section{Interpretation of proteomic analysis}

The production of NulOs by " $\mathrm{Ca}$. Accumulibacter" was confirmed by lectin staining, the recent mass spectrometry survey (Kleikamp et al. 2020a), and the phylogenetic analysis. To understand also the metabolism of NulOs, the proteome of the "Ca. Accumulibacter"-enriched biomass was studied using mass spectrometry. Out of the complete list of identified proteins (Table S1), it was found that " $\mathrm{Ca}$. Accumulibacter" expressed neuraminic acid receptor, permease, and tripartite ATP-independent periplasmic (TRAP) transporter proteins (Table 3). Those three proteins are used by some pathogenic bacteria as a mechanism to decorate their surface molecules, such as capsule polysaccharides, lipopolysaccharides, or flagellum, with sialic acids scavenged from the host; i.e., an extracellular sialic acid molecule is captured via a receptor (i.e., neuraminic acid receptor) and transported through the plasma membrane into the cell via a transporter (e.g., TRAP transporter), linking the sialic acid to a glycoconjugate and finally embedding the glycoconjugate within the plasma membrane (Honma et al. 2015). This suggests the presence of a NulO-specific utilization/recycling system in the " $\mathrm{Ca}$. Accumulibacter" enrichment, similar to the one in pathogenic bacteria. Other enzymes involved in the utilization of NulOs were not found, such as sialidases or enzymes involved in the catabolism of NulOs.
Fig. 4 Distance-based neighborjoining tree of the NAB-2 sequences (nonulosonic acid synthase). Sequences from bacteria, archaea, and animals were used. Enzymes are grouped based on their predicted nonulosonic acid specificity (color shading). Letters (a-1) indicate the enzymes present in the different available genomes of " $\mathrm{Ca}$. Accumulibacter" as shown in Table 2

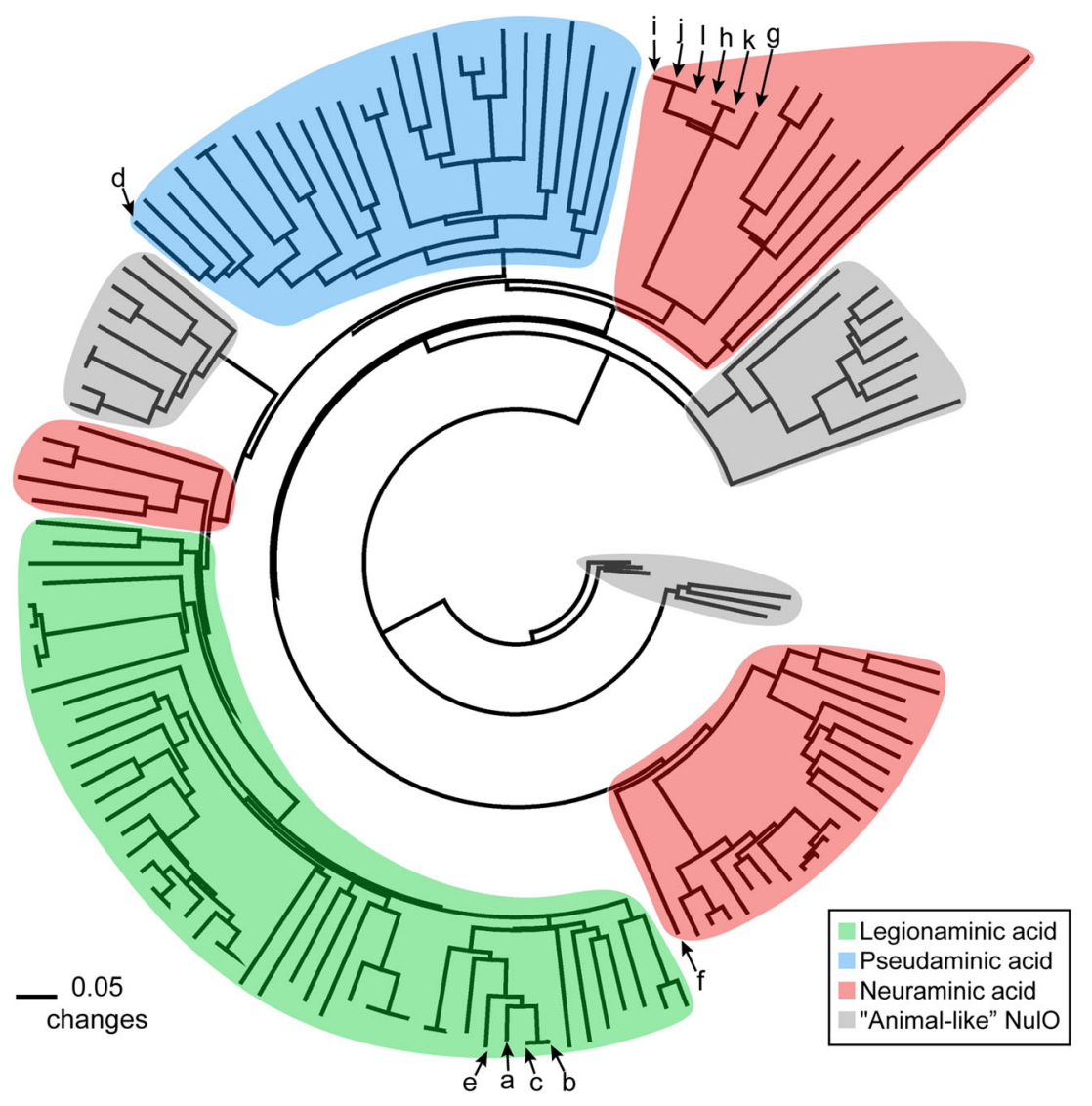


Table 3 Expressed proteins by "Ca. Accumulibacter" involved in the transport of NulOs. The complete list of identified proteins in the enriched biomass can be found in Table S1

\begin{tabular}{llll}
\hline Protein & Accession number & No. of unique peptides & Coverage (\%) \\
\hline Neu5Ac-binding protein & A0A011QNL6 & 11 & 58 \\
Neu5Ac permease & A0A011P7S7 & 4 & 6 \\
TRAP transporter solute receptor & A0A011QP03 & 3 & 73 \\
\hline
\end{tabular}

\section{Discussion}

\section{"Ca. Accumulibacter" and nonulosonic acid production}

Nonulosonic acids (NulOs) are a family of acidic carbohydrates with a nine-carbon backbone. They include sialic acids and other bacterial monosaccharides such as pseudaminic (Pse) and legionaminic (Leg) acids. NulOs have been observed at the surface of animal cells and pathogenic bacteria, but they have been generally overlooked in non-pathogenic microorganisms (Varki et al. 2017).

Recently, sialic acids were discovered in glycoproteins within the extracellular polymeric substances (EPS) of seawater-adapted aerobic granular sludge (de Graaff et al. 2019). "Ca. Accumulibacter" was suggested to be responsible for sialic acid production. However, to prove the link between the specific microorganism and sialic acid production, a study on a highly enriched culture was necessary. The granular biomass used in this study was proven to be highly enriched in " $\mathrm{Ca}$. Accumulibacter" (approx. 95\%, by proteomic investigations (Kleikamp et al. 2020b) and by FISH staining (Guedes da Silva et al. 2018)). Sialic acidspecific lectin staining displayed that sialic acids with $\alpha$ 2,3- and $\alpha$-2,6-linkage to the sub-terminal monosaccharide were distributed widely on the cell surface of " $\mathrm{Ca}$. Accumulibacter". In fact, these sialic acids visualized by lectin staining consist of diverse NulOs, i.e., Kdn (a common sialic acid) and Pse/Leg (bacterial NulOs) with various modifications, as the actual structure cannot be determined by lectin staining (Song et al. 2011). The most conserved enzyme (nonulosonic acid synthase, NAB-2) of the $\mathrm{NulO}$ biosynthetic pathway can be traced back from the available genomes of " $\mathrm{Ca}$. Accumulibacter". The lack of this enzyme in some genomes might be attributed to a lowquality or an incomplete state of those genomes and also to the genetic inability to produce NulOs of some genotypes of " $\mathrm{Ca}$. Accumulibacter". Phylogenetic analysis based on different sequences of the NAB-2 enzyme predicted the capacity of " $\mathrm{Ca}$. Accumulibacter" to produce Pse, Leg, and/or Neu (including Kdn). Therefore, this study provides evidence that " $\mathrm{Ca}$. Accumulibacter" can synthesize sialic acids and other NulOs. Moreover, it shows the significant diversity of NulOs available in biological environments, in addition to the most common sialic acid Neu5Ac.

\section{Importance of sialic acids and other nonulosonic acids in non-pathogenic bacteria}

The ability of bacteria to synthesize sialic acids has been mainly studied in a number of pathogens, where sialic acids or NulOs serve as a way of abolishing the immune response of the host by molecular mimicry (Carlin et al. 2009). Three different types of NulOs are frequently reported as produced by pathogenic bacteria: NeuAc, Pse, and Leg. Most NulOproducing pathogens synthesize one type of NulOs; e.g., Pasteurella multocida can synthesize NeuAc, Pseudomonas aeruginosa can synthesize Pse, and Clostridium botulinum can synthesize Leg. Only few pathogens, such as Campylobacter jejuni and Vibrio vulnificus can synthesize multiple types of NulOs depending on the strain examined (Almagro-Moreno and Boyd 2010). The production of NulOs confers specific advantages to these bacteria in the host-pathogen interaction. Surprisingly, " $\mathrm{Ca}$. Accumulibacter", a non-pathogenic bacterium that was cultivated in a bioreactor without any host-pathogen interaction, was found to produce different types of NulOs. Moreover, these compounds were also present in most bacteria and archaea recently tested by Kleikamp et al. (2020a). Thus, the synthesis of NulOs is not necessarily connected with the hostpathogen interaction.

Apart from the synthesis of NulOs, mammalian commensal and pathogenic bacteria that colonize sialic acid-rich tissues, such as the respiratory or the gastrointestinal tract, use hostderived sialic acids as competitive advantage. These bacteria take up sialic acids released from the host by means of dedicated transporters, either incorporating them into their cell surface macromolecules or metabolizing them as a source of carbon, nitrogen, and energy source (Almagro-Moreno and Boyd 2010). These bacteria that uptake/utilize sialic acids are closely associated with the host and exposed to a sialic acid-rich environment. However, it is extremely interesting to see that "Ca. Accumulibacter", cultivated without any NulOs in the media, still expressed Neu receptor, permease, and TRAP transporter proteins, which are essential for the uptake of NulOs. Therefore, the common understanding that both the 
abilities to synthesize and utilize NulOs are limited within pathogenic and/or commensal bacteria is not correct. These abilities might be widely spread in bacteria.

Most of the studies of NulOs to date have been focused on Neu5Ac, since it is the most abundant one in mammals (especially in humans). The findings that there were multiple NulOs produced by " $\mathrm{Ca}$. Accumulibacter" enrichment and microorganisms in seawater-adapted aerobic granules, together with other findings reported in literature, suggest the need to extend the consideration of NulOs beyond Neu5Ac alone when bacteria are involved.

\section{Nonulosonic acids and the EBPR process}

To avoid eutrophication due to phosphorus pollution, inorganic phosphorus is removed from wastewater by a process called EBPR. " $\mathrm{Ca}$. Accumulibacter" has been identified as the dominant organism responsible for EBPR (Zilles et al. 2002). This microorganism has been well studied in the past decades: Different genomic, proteomic, metabolic, and modelling studies are available (Oehmen et al. 2010; Barr et al. 2016; Oyserman et al. 2016; Guedes da Silva et al. 2018; Guedes da Silva et al. 2019; Rubio-Rincón et al. 2019). However, most of the studies overlook the extracellular matrix.

Sialic acids are known to participate in cell-cell interactions in mammals (Schnaar et al. 2014). Especially due to their electronegative nature, together with their bulky structure, they form a protective layer surrounding the cell. NulOs might play a similar role in "C $\mathrm{a}$. Accumulibacter" and provide advantages in competing with other microorganisms in the EBPR process. " $\mathrm{Ca}$. Accumulibacter" is able to synthesize multiple NulOs with various modifications. These structures cannot be recognized by a single type of sialidase (as shown in the enzymatic analysis); therefore, they can protect the cells from enzymatic degradation. On the other hand, when NulOs become available (e.g., released from their own macromolecules), the expression of specific transporters by " $\mathrm{Ca}$. Accumulibacter" allows them to re-uptake these carbohydrates and re-utilize them, avoiding synthesizing them de novo. Through this recycling, less nutrients and cellular energy resources are required, perhaps this strategy provides " $\mathrm{Ca}$. Accumulibacter" advantages in competition as well. Moreover, in vertebrates, sialic acids are typically found as terminal residues on the glycan chains of extracellular glycoconjugates, acting as "bridging" molecules between cells, and between cells and extracellular matrices (Chen and Varki 2010). Although NulOs have been overlooked in environmental bacteria, their known roles as recognition molecules in other organisms suggest that they may be involved in functions such as regulation of " $\mathrm{Ca}$. Accumulibacter" bioaggregate formation in wastewater treatment process and natural estuarine systems or mediating recognition for bacteriophages. Therefore, sialic acids and other nonulosonic acids should be investigated in further detail to understand their role in the ecology of "Ca. Accumulibacter" and even in the EBPR process in particular, and biofilms in general.

Supplementary Information The online version contains supplementary material available at https://doi.org/10.1007/s00253-021-11249-3.

Acknowledgements The authors would like to thank Leonor Guedes da Silva and Danny de Graaff for providing the biomass used in these investigations, and Ute Kuhlicke for her help with the FLBA-CLSM analyses and digital image processing.

Code availability Not applicable

Author contribution STM and YL planned the research based on intensive discussions among all the authors, especially with MvL and DW. MP and $\mathrm{HK}$ conducted the mass spectrometry analyses. TN performed the fluorescence lectin-binding analysis. STM worked on the phylogenetic analysis and additional lab work. STM and YL interpreted the data and played major roles in drafting, writing, and revising the manuscript. All authors read and approved the manuscript.

Funding This work is part of the research project "Nature inspired biopolymer nanocomposites towards a cyclic economy" (Nanocycle) funded by the programme Closed cycles - Transition to a circular economy (grant no. ALWGK.2016.025) of the Earth and Life Sciences Division of the Dutch Research Council (NWO).

Data availability The data generated and/or analyzed during the current study are included in this article and its supplementary material.

\section{Declarations}

Ethical approval Not applicable

Consent to participate Not applicable

Consent for publication Not applicable

Conflict of interest The authors declare no competing interests.

Open Access This article is licensed under a Creative Commons Attribution 4.0 International License, which permits use, sharing, adaptation, distribution and reproduction in any medium or format, as long as you give appropriate credit to the original author(s) and the source, provide a link to the Creative Commons licence, and indicate if changes were made. The images or other third party material in this article are included in the article's Creative Commons licence, unless indicated otherwise in a credit line to the material. If material is not included in the article's Creative Commons licence and your intended use is not permitted by statutory regulation or exceeds the permitted use, you will need to obtain permission directly from the copyright holder. To view a copy of this licence, visit http://creativecommons.org/licenses/by/4.0/.

\section{References}

Almagro-Moreno S, Boyd EF (2010) Bacterial catabolism of nonulosonic (sialic) acid and fitness in the gut. Gut Microbes 1:45-50. https://doi. org/10.4161/gmic.1.1.10386 
Angata T, Varki A (2002) Chemical diversity in the sialic acids and related $\alpha$-keto acids: An evolutionary perspective. Chem Rev 102: 439-469. https://doi.org/10.1021/cr000407m

Barr JJ, Dutilh BE, Skennerton CT, Fukushima T, Hastie ML, Gorman JJ, Tyson GW, Bond PL (2016) Metagenomic and metaproteomic analyses of Accumulibacter phosphatis-enriched floccular and granular biofilm. Environ Microbiol 18:273-287. https://doi.org/10.1111/ 1462-2920.13019

Bennke CM, Neu TR, Fuchs BM, Amann R (2013) Mapping glycoconjugate-mediated interactions of marine Bacteroidetes with diatoms. Syst Appl Microbiol 36:417-425. https://doi.org/10.1016/ j.syapm.2013.05.002

Boleij M, Pabst M, Neu TR, van Loosdrecht MCM, Lin Y (2018) Identification of glycoproteins isolated from extracellular polymeric substances of full-scale anammox granular sludge. Environ Sci Technol 52:13127-13135. https://doi.org/10.1021/acs.est.8b03180

Boleij M, Seviour T, Wong LL, van Loosdrecht MCM, Lin Y (2019) Solubilization and characterization of extracellular proteins from anammox granular sludge. Water Res 164:114952. https://doi.org/ 10.1016/J.WATRES.2019.114952

Carlin AF, Uchiyama S, Chang YC, Lewis AL, Nizet V, Varki A (2009) Molecular mimicry of host sialylated glycans allows a bacterial pathogen to engage neutrophil Siglec-9 and dampen the innate immune response. Blood 113:3333-3336. https://doi.org/10.1182/ blood-2008-11-187302

Chen X, Varki A (2010) Advances in the biology and chemistry of sialic acids. ACS Chem Biol 5:163-176

de Graaff DR, Felz S, Neu TR, Pronk M, van Loosdrecht MCM, Lin Y (2019) Sialic acids in the extracellular polymeric substances of seawater-adapted aerobic granular sludge. Water Res 155:343351. https://doi.org/10.1016/J.WATRES.2019.02.040

Deng L, Chen X, Varki A (2013) Exploration of sialic acid diversity and biology using sialoglycan microarrays. Biopolymers 99:650-665

Felz S, Al-Zuhairy S, Aarstad OA, van Loosdrecht MCM, Lin YM (2016) Extraction of structural extracellular polymeric substances from aerobic granular sludge. J Vis Exp e54534 . https://doi.org/ $10.3791 / 54534$

Felz S, Vermeulen P, van Loosdrecht MCM, Lin YM (2019) Chemical characterization methods for the analysis of structural extracellular polymeric substances (EPS). Water Res 157:201-208. https://doi. org/10.1016/J.WATRES.2019.03.068

Flemming H-C, Wingender J (2010) The biofilm matrix. Nat Rev Microbiol 8:623-633. https://doi.org/10.1038/nrmicro2415

Gallagher JT, Morris A, Dexter TM (1985) Identification of two binding sites for wheat-germ agglutinin on polylactosamine-type oligosaccharides. Biochem J 231:115-122. https://doi.org/10.1042/ bj2310115

Guedes da Silva L (2020) Life in changing environments: The intriguing cycles of polyphosphate accumulating organisms. Dissertation. Delft University of Technology

Guedes da Silva L, Gamez KO, Gomes JC, Akkermans K, Welles L, Abbas B, van Loosdrecht MCM, Wahl SA (2018) Revealing metabolic flexibility of Candidatus Accumulibacter phosphatis through redox cofactor analysis and metabolic network modeling. bioRxiv 86:458331. https://doi.org/10.1101/458331

Guedes da Silva L, Tomás-Martínez S, van Loosdrecht MCM, Wahl SA (2019) The environment selects: Modeling energy allocation in microbial communities under dynamic environments. bioRxiv 689174 . https://doi.org/10.1101/689174

Hanisch F, Weidemann W, Großmann M, Joshi PR, Holzhausen HJ, Stoltenburg G, Weis J, Zierz S, Horstkorte R (2013) Sialylation and muscle performance: Sialic acid is a marker of muscle ageing. PLoS One 8:e80520. https://doi.org/10.1371/journal.pone.0080520

Honma K, Ruscitto A, Frey AM, Stafford GP, Sharma A (2015) Sialic acid transporter NanT participates in Tannerella forsythia biofilm formation and survival on epithelial cells. Microb Pathog 94:12-20. https://doi.org/10.1016/j.micpath.2015.08.012

Kleikamp HBC, Lin YM, McMillan DGG, Geelhoed JS, Naus-Wiezer SNH, van Baarlen P, Saha C, Louwen R, Sorokin DY, van Loosdrecht MCM, Pabst M (2020a) Tackling the chemical diversity of microbial nonulosonic acids - A universal large-scale survey approach. Chem Sci 11:3074-3080. https://doi.org/10.1039/ C9SC06406K

Kleikamp HBC, Pronk M, Tugui C, Guedesda Silva L, Abbas B, Mei Lin Y, van Loosdrecht MCM, Pabst M (2020b) Quantitative profiling of microbial communities by 2 de novo metaproteomics. bioRxiv 2020:2020.08.16.252924. https://doi.org/10.1101/2020.08.16. 252924

Knibbs RN, Goldstein IJ, Ratcliffe RM, Shibuya N (1991) Characterization of the carbohydrate binding specificity of the leukoagglutinating lectin from Maackia amurensis. Comparison with other sialic acid-specific lectins. J Biol Chem 266:83-88

Knirel YA, Shashkov AS, Tsvetkov YE, Jansson PE, Zähringer U (2003) 5,7-Diamino-3,5,7,9-tetradeoxynon-2-ulosonic acids in bacterial glycopolymers: Chemistry and biochemistry. Adv Carbohydr Chem Biochem 58:371-417. https://doi.org/10.1016/S00652318(03)58007-6

Lambre CR, Kazatchkine MD, Maillet F, Thibon M (1982) Guinea pig erythrocytes, after their contact with influenza virus, acquire the ability to activate the human alternative complement pathway through virus-induced desialation of the cells. J Immunol 128: 629-634

Lewis AL, Desa N, Hansen EE, Knirel YA, Gordon JI, Gagneux P, Nizet $\mathrm{V}$, Varki A (2009) Innovations in host and microbial sialic acid biosynthesis revealed by phylogenomic prediction of nonulosonic acid structure. Proc Natl Acad Sci U S A 106:13552-13557. https:// doi.org/10.1073/pnas.0902431106

Mainstone CP, Parr W (2002) Phosphorus in rivers - Ecology and management. Sci Total Environ 282-283:25-47. https://doi.org/10. 1016/S0048-9697(01)00937-8

Neu T, Kuhlicke U (2017) Fluorescence lectin bar-coding of glycoconjugates in the extracellular matrix of biofilm and bioaggregate forming microorganisms. Microorganisms 5:5. https://doi.org/10.3390/microorganisms5010005

Neu TR, Lawrence JR (2016) Laser microscopy for the study of biofilms: Issues and options. In: Romani AM, Guasch H, Balaguer MD (eds) Aquatic biofilms: Ecology, water quality and wastewater treatment. Caister Academic Press, Norfolk, pp 29-46

Neu TR, Lawrence JR (2017) The extracellular matrix - An intractable part of biofilm systems. In: Flemming H-C, Neu TR, Wingender J (eds) The perfect slime - Microbial extracellular polymeric substances (EPS). IWA Publishing, London, pp 25-60

Oehmen A, Lopez-Vazquez CM, Carvalho G, Reis MAM, van Loosdrecht MCM (2010) Modelling the population dynamics and metabolic diversity of organisms relevant in anaerobic/anoxic/aerobic enhanced biological phosphorus removal processes. Water Res 44:4473-4486. https://doi.org/10.1016/j.watres.2010.06.017

Oyserman BO, Noguera DR, del Rio TG, Tringe SG, McMahon KD (2016) Metatranscriptomic insights on gene expression and regulatory controls in Candidatus Accumulibacter phosphatis. ISME J 10: 810-822. https://doi.org/10.1038/ismej.2015.155

Petit D, Teppa E, Cenci U, Ball S, Harduin-Lepers A (2018) Reconstruction of the sialylation pathway in the ancestor of eukaryotes. Sci REPORTS 8:2946. https://doi.org/10.1038/s41598-01820920-1

Ravindranath MH, Higa HH, Cooper EL, Paulson JC (1985) Purification and characterization of an $\mathrm{O}$-acetylsialic acid-specific lectin from a marine crab Cancer antennarius. J Biol Chem 260:8850-8856

Rubio-Rincón FJ, Weissbrodt DG, Lopez-Vazquez CM, Welles L, Abbas B, Albertsen M, Nielsen PH, van Loosdrecht MCM, Brdjanovic D (2019) "Candidatus Accumulibacter delftensis": A clade IC novel 
polyphosphate-accumulating organism without denitrifying activity on nitrate. Water Res 161:136-151. https://doi.org/10.1016/J. WATRES.2019.03.053

Schnaar RL, Gerardy-Schahn R, Hildebrandt H (2014) Sialic acids in the brain: Gangliosides and polysialic acid in nervous system development, stability, disease, and regeneration. Physiol Rev 94:461-518. https://doi.org/10.1152/physrev.00033.2013

Seviour RJ, Mino T, Onuki M (2003) The microbiology of biological phosphorus removal in activated sludge systems. FEMS Microbiol Rev 27:99-127

Seviour T, Derlon N, Dueholm MS, Flemming H-C, Girbal-Neuhauser E, Horn H, Kjelleberg S, van Loosdrecht MCM, Lotti T, Malpei MF, Nerenberg R, Neu TR, Paul E, Yu H, Lin Y (2019) Extracellular polymeric substances of biofilms: Suffering from an identity crisis. Water Res 151:1-7. https://doi.org/10.1016/J.WATRES.2018.11. 020

Shibuya N, Goldstein IJ, Broekaert WF, Nsimba-Lubaki M, Peeters B, Peumans WJ (1987) The elderberry (Sambucus nigra L.) bark lectin recognizes the Neu5Ac(alpha 2-6)Gal/GalNAc sequence. J Biol Chem 262:1596-1601

Soares RMA, Rosangela RM, Alviano DS, Angluster J, Alviano CS, Travassos LR (2000) Identification of sialic acids on the cell surface of Candida albicans. Biochim Biophys Acta, Gen Subj 1474:262268. https://doi.org/10.1016/S0304-4165(00)00003-9

Song X, Yu H, Chen X, Lasanajak Y, Tappert MM, Air GM, Tiwari VK, Cao H, Chokhawala HA, Zheng H, Cummings RD, Smith DF (2011) A sialylated glycan microarray reveals novel interactions of modified sialic acids with proteins and viruses. J Biol Chem 286: 31610-31622. https://doi.org/10.1074/jbc.M111.274217

Traving C, Schauer R (1998) Structure, function and metabolism of sialic acids. Cell Mol Life Sci 54:1330-1349

Varki A, Schnaar RL, Schauer R (2017) Sialic acids and other nonulosonic acids. In: Varki A (ed) Essentials of glycobiology, 3rd edn. Cold Spring Harbor Laboratory Press, Cold Spring Harbor (NY), pp 179-195

Watson SJ, Needoba JA, Peterson TD (2019) Widespread detection of Candidatus Accumulibacter phosphatis, a polyphosphateaccumulating organism, in sediments of the Columbia River estuary. Environ Microbiol 21:1369-1382. https://doi.org/10.1111/14622920.14576

Weissbrodt DG, Neu TR, Kuhlicke U, Rappaz Y, Holliger C (2013) Assessment of bacterial and structural dynamics in aerobic granular biofilms. Front Microbiol 4. https://doi.org/10.3389/fmicb.2013. 00175

Zilles JL, Peccia J, Kim MW, Hung CH, Noguera DR (2002) Involvement of Rhodocyclus-related organisms in phosphorus removal in full-scale wastewater treatment plants. Appl Environ Microbiol 68:2763-2769. https://doi.org/10.1128/AEM.68.6.27632769.2002

Publisher's note Springer Nature remains neutral with regard to jurisdictional claims in published maps and institutional affiliations. 